\title{
Contextual dependencies in motor skills
}

\author{
DAVID L. WRIGHT and CHARLES H. SHEA \\ Texas A\& M University, College Station, Texas
}

\begin{abstract}
The development of contextual dependencies during motor skill acquisition was examined. Environmental context was varied along intentional and incidental dimensions. Intentional stimuli were defined as essential for achieving skilled performance, whereas incidental stimuli were defined as those that have the potential to become associated with specific tasks due to their selective presence in the learning environment. Experiment 1 demonstrated the occurrence of contextual dependencies for the learning of four-key typing sequences. Contextual dependencies were diminished in Experiment 2 when the number of keys used in the sequences was reduced. In Experiment 3, a retention condition was incorporated, in which both the intentional and the incidental stimuli were not available; this confirmed that task difficulty mediated the development of contextual dependencies. These findings are discussed with respect to the incorporation of environmental contextual stimuli with memorial representations of movement information.
\end{abstract}

Almost everyone has stepped away from a desk in order to do something and before reaching the destination has forgotten what it was that he or she intended to do. The solution for many individuals is to return to the desk in the hope that the surroundings will assist in the reinstatement or retrieval of the memory. This is an example of context-dependent memory, a phenomenon in which cognitive processing is affected in subtle, and ofien important, ways by the environmental context in which an experience occurs (see S. M. Smith, 1988 for review). Although environmental context has been defined in a number of ways (Eich, Weingartner, Stillman, \& Gillin, 1975; S. M. Smith, Glenberg, \& Bjork, 1978), for the present purpose, environmental stimuli will be classified along intentional and incidental diménsions. Intentional stimuli are those that are explicitly identified as essential to task acquisition, and incidental stimuli are those that have the potential to become associated with a specific task because of their selective presence in the learning environment: ${ }^{1}$

When a person acquires a motor task, often one or more intentional stimuli in the learning environment can be used in the reliable execution of an appropriate response. For example, while learning to drive a standard shift car, a beginning driver may attend to the tachometer and/or the speedometer in order to determine the moment at which a gear change should occur. However, if the environmental context is examined closely, there are usually a number of environmental stimuli that, to varying degrees, coincide with the presence of the intentional stimuli. It

The authors would like to thank Eric Eich, Tim Lee, Robert Proctor, and Steve Smith for reading an earlier draft of this paper and contributing many helpful suggestions for revision. In addition, we wish to thank Robin Loy, Yuhua Li, and Chad Whiticare for their assistance with data collection. Requests for reprints should be addressed to David Wright, Human Performance Laboratories, 276 Read Building, Texas A\& M University, College Station, TX 77843-4243. is these stimuli that are herein considered incidental. Thus, it is possible, as the learning of a task progresses, that the performer of the task will begin to simultaneously process some subset of the incidental stimuli along with the intentional stimuli. To extend the driving examplethe novice driver may begin to associate engine sounds, vibrations, acceleration cues, or road sounds with the need to shift gears, even though no explicit instruction has been given to do so.

As long as the incidental stimuli maintain a stable relationship with the intentional stimuli, it is possible that an individual may utilize this information to execute an appropriate response (Underwood, Ham, \& Ekstrand, 1962). In many cases, however, there may only be a spurious relationship between intentional and incidental stimuli. Should the relationship between the incidental and intentional stimuli change or be eliminated at any stage, the association developed between intentional and incidental stimuli may prove to be problematic. In the example of driving, changes in road surface or terrain may cause the relationship between one or more of the incidental stimuli and the intentional stimuli (i.e., monitoring the speedometer) to be altered. At this point, any reliance on such relationships might prove detrimental to performance.

In the verbal domain, it has been demonstrated that recall and relearning performance improves if the subject is tested in the original learning environment, rather than in an environment that has been changed (Reed, 1931; S. Smith \& Guthrie, 1924). These findings suggest that as particular skills are learned, memorial representations incorporate parts of the original learning context that can subsequently be used as retrieval cues for accessing necessary task-specific information. It has been suggested that contextual dependencies may also influence the learning of motor skills. Lintern (1985) demonstrated that enriching the contextual environment during the learning of an aircraft landing simulation task 
(by providing additional visual cues) aided task acquisition and transfer. Lintern noted, however, that if the provision of the additional cues continued over an extended period of practice, the probability of a dependency on this information increased. She also suggested that, should this dependency emerge, it might ultimately result in negating the potential benefits of the additional cues. On a practical note, the role of contextual dependencies may also resemble and offer important implications regarding the "home-court advantage" phenomenon that has previously been examined in the sport psychology literature (Schwartz \& Barsky, 1977; Silva, Andrew, \& Richey, 1983; Varca, 1980).

We designed the present set of experiments to address the question of whether or not contextual dependencies develop in the motor domain. Of primary interest is the question of whether or not contextual reinstatement is as potent in motor skill learning as it has been demonstrated to be for verbal skill learning (S. M. Smith, 1988). In three experiments, we attempted to determine the effect of the execution of motor tasks in a context in which we either switched or maintained the same intentional and incidental stimuli that subjects had experienced during acquisition. In each experiment, the subjects practiced three sequences of keystrokes in a particular acquisition context. Their retention was subsequently tested either in the same or in a different context. The subjects acquired sequences involving four keystrokes in Experiment 1, and sequences involving three keystrokes in Experiment 2. Experiment 3 was a direct comparison of performance on the three- and four-key tasks, in which the memorability of the sequences was determined when neither the intentional nor the incidental stimuli were available to aid subjects' recall.

\section{EXPERIMENT 1}

In Experiment 1, we investigated whether or not retention of motor skills (three sequences of four key strokes) is facilitated by environmental contextual reinstatement. More specifically, a particular subset of intentional and incidental stimuli was chosen to be manipulated during the retention phase of Experiment 1. After a period of acquisition, the subjects were required to reproduce the acquired key sequences with (1) the same intentional/same incidental context as originally practiced (contextual reinstatement), (2) the same intentional/switched incidental context, or (3) no intentional/same incidental context.

This is similar to the protocol used by Watkins, Ho, and Tulving (1976) to investigate facial recognition. They demonstrated that altering the associations that had developed during acquisition resulted in performance decrements at retention (also see Underwood et al., 1962, who used a paired associate task and cued recall). If associations between incidental and intentional stimuli are developed, then to remove the intentional stimuli or to switch the relationship between the intentional and incidental stimuli should cause a greater retention decrement than should a condition in which the original acquisition context is reinstated.

\section{Method}

Subjects. The subjects were 45 undergraduate students from Texas A \& M University who received class credit for their participation.

Apparatus. The apparatus consisted of an IBM-compatible 286 microcomputer with EGA color monitor and standard keyboard. The microcomputer was programmed to scan the keyboard 2,000 times per second.

Procedures. The subjects entered a testing room, where they were seated facing a computer monitor and asked to place their fingers on the keyboard in a normal touch-typing position (" $a$," " $s$, , "d," " f " for the left hand and " $j$, ," "k," "l," "“;" for the right hand). The subjects were informed that a display would appear on the monitor, indicating a sequence of keys that they should press-which fingers (keys) they should use, and the order in which they should do this. The three sequences that were used are shown in Figure 1. Each sequence required the use of the same four fingers (left little, left index, right middle, and right ring) but in a different order. The subjects were not informed that only four fingers would be utilized or that only three sequences would be presented.

Each display provided both intentional and incidental stimuli. The intentional stimuli were the numbers that indicated the appropriate

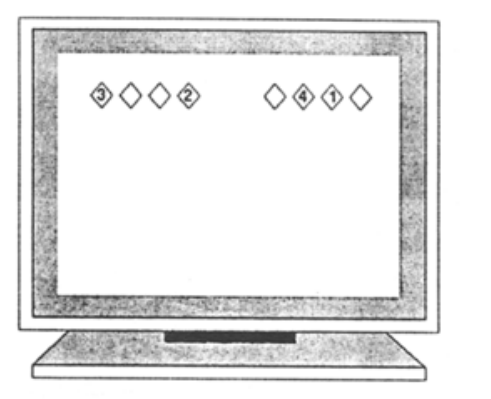

POSITION $=$ TOP COLOR $=$ BLUE SOUND $=2500 \mathrm{HZ}$ SHAPE $=$ DIAMOND

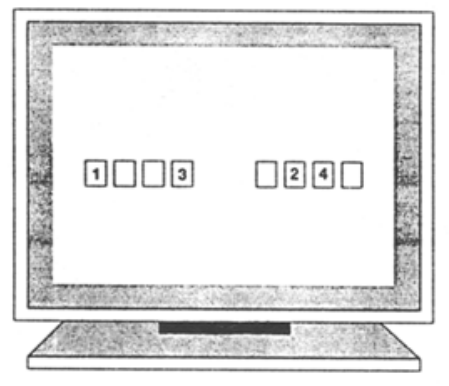

POSITION $=$ MIDDLE COLOR $=$ RED SOUND $=1000 \mathrm{HZ}$ SHAPE $=$ SQUARE

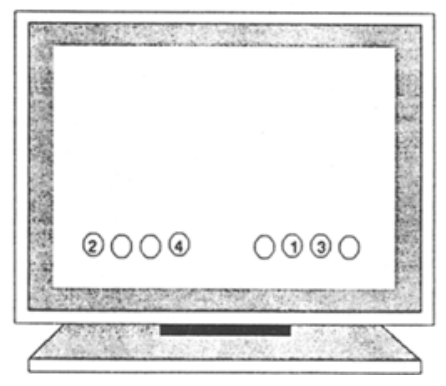

POSITION = BOTTOM COLOR $=$ YELLOW SOUND $=300 \mathrm{HZ}$ SHAPE $=$ CIRCLE
Figure 1. Graphics screen displays for the three sequences in Experiment 1. Note that each sequence was colored and accompanied by a tone. 
keys and sequence. The incidental stimuli consisted of the color of the display (blue, red, or yellow), the shape of the key designations (diamond, square, or circle), a tone generated during presentation $(2500,1000$, or $300 \mathrm{~Hz})$, and the position on the screen (top, middle, or bottom row). The subjects were told to use the intentional stimuli to plan and execute the sequence of keypresses, but no mention was made of the incidental stimuli. Intentional and incidental stimuli were consistently mapped throughout acquisition so that each sequence was always presented in the same color, shape, and tone, and in the same position on the monitor.

Retention performance was evaluated in one of three conditions. In the same intentional/same incidental condition, the context established during acquisition was reinstated. In the same intentional/switched incidental condition, intentional stimuli were presented along with a set of incidental stimuli that had previously been paired with alternative intentional stimuli during acquisition. Finally, in the no intentional/same incidental condition, a set of incidental stimuli was presented in the absence of any intentional stimuli.

At the beginning of each trial, a display appeared on the computer monitor for a brief period of time $(400,600$, or $800 \mathrm{msec})$. The subjects were instructed to initiate a sequence only after the display was removed from the computer monitor. They were told that the sequence of keypresses should be completed within $2 \mathrm{sec}$. On completion of a trial, the subjects received feedback that indicated the success or failure on that particular trial. The message "good trial" appeared when the subject typed the appropriate sequence within the specified time limit. "Bad trial" appeared when the subject began the sequence too early, completed the sequence after the 2-sec time limit, or typed the wrong sequence.

All subjects were administered nine acquisition blocks of 12 trials. Each trial block contained four presentations of each of the sequences. Sequence presentations were ordered within each block so that no sequence was performed on more than two consecutive trials. Trial blocks were separated by a $20-\mathrm{sec}$ rest intervals. The retention trial block consisted of 3 trials ( 1 of each sequence) presented $20 \mathrm{sec}$ after the completion of the last acquisition trial block.

On completion of the experiment, verbal reports were obtained from the subjects regarding the extent to which they noticed any contingencies between intentional stimuli, incidental stimuli, and key sequences. The verbal reports were initially prompted by openended questions. If the subjects did not report relationships between incidental and intentional stimuli, the questions became more explicit. Each test session (acquisition and retention) lasted approximately $30 \mathrm{~min}$

\section{Results}

Performance was evaluated in terms of percent correct responses. A correct response was defined as the subject's pressing the appropriate keys in the correct order within the 2-sec time limit. Acquisition and retention performance for all conditions are illustrated in Figure 2, as a function of trial block. Note that acquisition performance has been collapsed across retention conditions.

Acquisition. All subjects experienced the same conditions during acquisition. However, a retention condition $X$ acquisition block analysis of variance (ANOVA) with repeated measures on the last factor was performed to determine whether random assignment to groups had resulted in differences across acquisition blocks. The analysis failed to indicate either a main effect of retention condition $[F(2,42)=0.62, p>.05]$ or a retention condition $\times$ acquisition block interaction $[F(16,336)=1.1$, $p>.05]$. The analysis did indicate a main effect of acquisition block $[F(8,336)=42.83, p<.001]$. Duncan's New Multiple Range Test indicated that Acquisition Blocks 1-4 were different from each other, with no reliable differences thereafter.

Retention. A retention condition ANOVA indicated a main effect of retention condition $[F(2,42)=10.86$, $p<.001]$. Duncan's New Multiple Range Test revealed that subjects tested in the same intentional/same incidental retention condition $(M=13.8 \%)$ performed with less error than did subjects tested in the same intentional/switched incidental retention condition $(M=$ $52.3 \%)$. Absence of the intentional stimuli $(M=75.0 \%)$

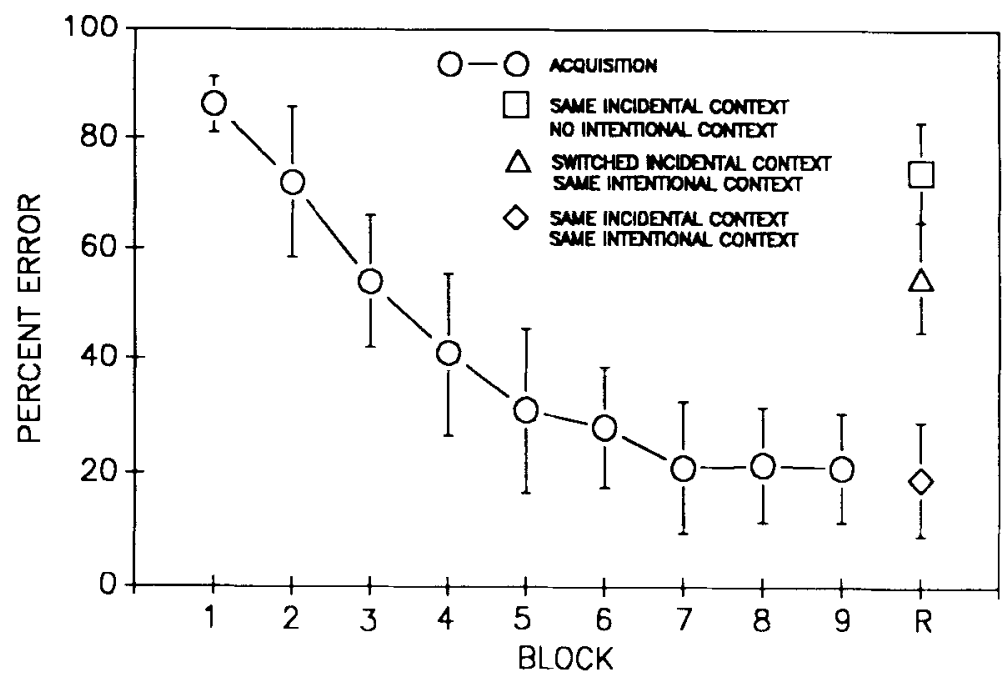

Figure 2. Acquisition and retention performance for the nine acquisition blocks and one retention block in Experiment 1. Note that acquisition performance has been collapsed across retention conditions. Acquisition błocks represent an average of 12 trials, while the retention block is an average of 3 trials. 
was found to further increase error in performance during retention.

Thus, changes of either the intentional or the incidental stimuli at retention negatively affected retention performance. However, the subjects did not appear to be aware, at least to the extent necessary for verbalization, of the relationships that existed between the intentional stimuli, the incidental stimuli, and the sequences. Even when the subjects were prompted regarding the association between the key sequences and each color, tone, key shape, and display position, they were unable to verbalize the relationships or specify what conditions and/or stimuli had been changed during retention testing.

\section{Discussion}

Switching the relationship between the intentional and incidental stimuli from that established in the original learning environment had a detrimental effect on the subsequent performance of the keypress sequences. In addition, removing the intentional cues and requiring an individual to perform the keypress task in the presence of the incidental stimuli alone resulted in a further decrement in retention performance. These findings are consistent with those from studies of verbal learning, which indicate superior recall of material when retention performance is required in the original learning environment (S. M. Smith, 1988). Thus, they indicate that motor skill learning is influenced by contextual dependencies that develop during task acquisition. Even though an individual can be directed to attend to particular event information (intentional stimuli) in order to aid task acquisition, a degree of dependency on other contextual information (incidental stimuli) can develop.

Experiencing the same intentional/switched incidental context at retention led to errors in producing an appropriate key sequence. This indicates the development of a contextual dependency due to the emergence of an association between the intentional and the incidental stimuli. Furthermore, the poor performance exhibited when only the incidental stimuli were available at retention suggests a weak relationship, at best, between the incidental stimuli and each of the tasks. The fact that subjects were unable to verbalize these associations supports this contention. Figure 3 is a simplified diagram of the associations proposed to have developed during acquisition.

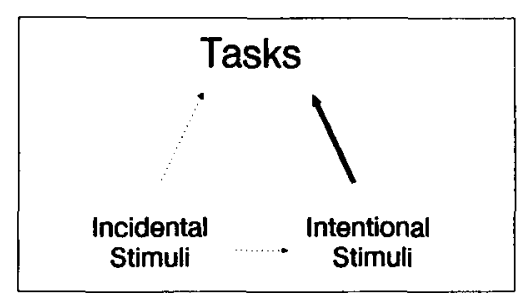

Figure 3. Simplified diagram illustrating the associations proposed to have been developed for the four-key task as a result of acquisition exposures.
It should be noted that the sequences utilized in Experiment 1 appeared to be very difficult for many of the subjects. At the end of acquisition, subjects exhibited in excess of $20 \%$ errors. Moreover, examination of the acquisition data for the same intentional/switched incidental retention context condition revealed an interesting trend. Subjects demonstrating the largest error during the last block of acquisition were disrupted by changes in the retention context more than the more proficient subjects were. $^{2}$ Alternatively, the subjects exhibiting the least error at the end of acquisition were less dependent on reinstatement of the original learning context. This suggests that the relative difficulty of the task may influence the degree to which contextual dependencies develop when subjects learn a motor skill. If difficulty is indeed a mediating variable for the development of contextual dependencies, learning a less difficult task may change the nature of the dependencies as a result of changes in the memorial associations that develop. To investigate this issue, we performed Experiment 2.

\section{EXPERIMENT 2}

Experiment 2 was an attempt to extend the findings of Experiment 1 by examining the extent to which the learning of an easier motor task would influence the development of contextual dependencies. It was possible that contextual dependencies might be reduced as the task to be learned became less complex. In the verbal domain, it had been demonstrated that reducing the difficulty of the memory task by increasing the meaningfulness of CVCs (Vela, 1984) or by categorizing (Eich et al., 1975) the words in a list tends to result in a reduction in contextual dependencies elicited at retention. The subjects in Experiment 2 learned three tasks, each of which required a different sequence of three keystrokes. The reduction in the number of keypresses required in the sequences was intended to decrease the difficulty of the tasks.

One explanation for contextual dependencies' being diminished as the task is reduced in difficulty is that there is a reduced need for processing the incidental cues to aid acquisition performance. If this is the case, for a relatively easy motor task the same intentional/switched incidental retention condition should result in little, if any, decrement in retention performance. Furthermore, this perspective would predict poor performance when retention is tested in the presence of the incidental stimuli alone, since these stimuli are assumed not to be processed during acquisition (see Figure 4, top).

However, an alternative proposal is that as practice on a relatively easy motor task progresses, subjects have the opportunity to more fully analyze relationships between stimuli (both intentional and incidental) and the task to be learned (see Figure 4, bottom). In this scenario, one would predict that the relationship between the incidental stimuli and the tasks would ultimately become apparent and be included as part of the internal representation of the task. This proposal predicts similar findings for the 

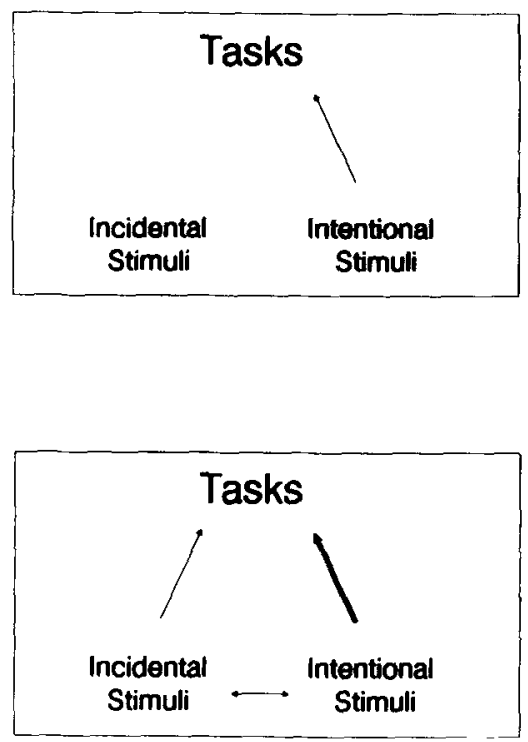

Figure 4. Simplified diagrams illustrating the associations that could develop for the three-key task as a result of acquisition exposures.

same intentional/switched incidental retention condition as does the previous alternative. That is, when the associations that have developed between the intentional and incidental stimuli are disrupted, performance should only suffer minimally, because the conflict between stimuli is easily resolved. In this case, the stronger, more developed relationship between the task and intentional stimuli would dominate over the weaker, less developed relationship between the tasks and the incidental stimuli. This proposal is reminiscent of the outshining principle that has been described in the verbal domain (see, e.g., S. M. Smith, 1986,1988 ). The outshining principle emphasizes the relative strength of environmental cues, with a better (stronger) cue outshining weaker cues. This suggests that, should a conflict in cues be encountered, the stronger, more developed cue(s) will be utilized.

However, for this alternative a different prediction would be made for the no intentional/same incidental retention condition. When the incidental stimuli are presented alone, the weaker association that has developed between incidental stimuli and the task might be adequate to support successful performance. Since the intentional stimuli are not present, the relationship between the incidental stimuli and the task would be allowed to surface.

\section{Method}

Subjects. The subjects were 45 undergraduate students from Texas A\& M University who received class credit for their participation.

Apparatus. The apparatus was identical to that in Experiment 1.

Procedures. The procedures were identical to those in Experiment 1 , with the exception of the number of keystrokes utilized in the three sequences. Figure 5 illustrates the presentation of the three sequences in Experiment 2; each sequence required the use of the same three fingers (left ring, left middle, and right little) but in a different order.

\section{Results}

Performance was evaluated in terms of percent correct responses. Acquisition and retention performance for all conditions are illustrated in Figure 6, as a function of trial block. Note that acquisition performance has been collapsed across retention conditions.

Acquisition. All subjects experienced the same conditions during acquisition. However, a retention condition $x$ acquisition block ANOVA with repeated measures on the last factor was performed to determine whether random assignment to groups resulted in differences across acquisition blocks. The analysis failed to indicate either a main effect of retention condition $[F(2,42)=2.82$, $p>.05]$ or a retention condition $\times$ acquisition block interaction $[F(16,336)=1.72, p>.05]$. The analysis did indicate a main effect of acquisition block $[F(8,336)=$ 46.93, $p<.01]$. Duncan's New Multiple Range Test indicated that Acquisition Blocks 1 and 2 were different from each other, with no difference thereafter.

Retention. Switching the incidental stimuli or withdrawing the intentional stimuli did not appear to influence retention performance. Indeed, a retention condition

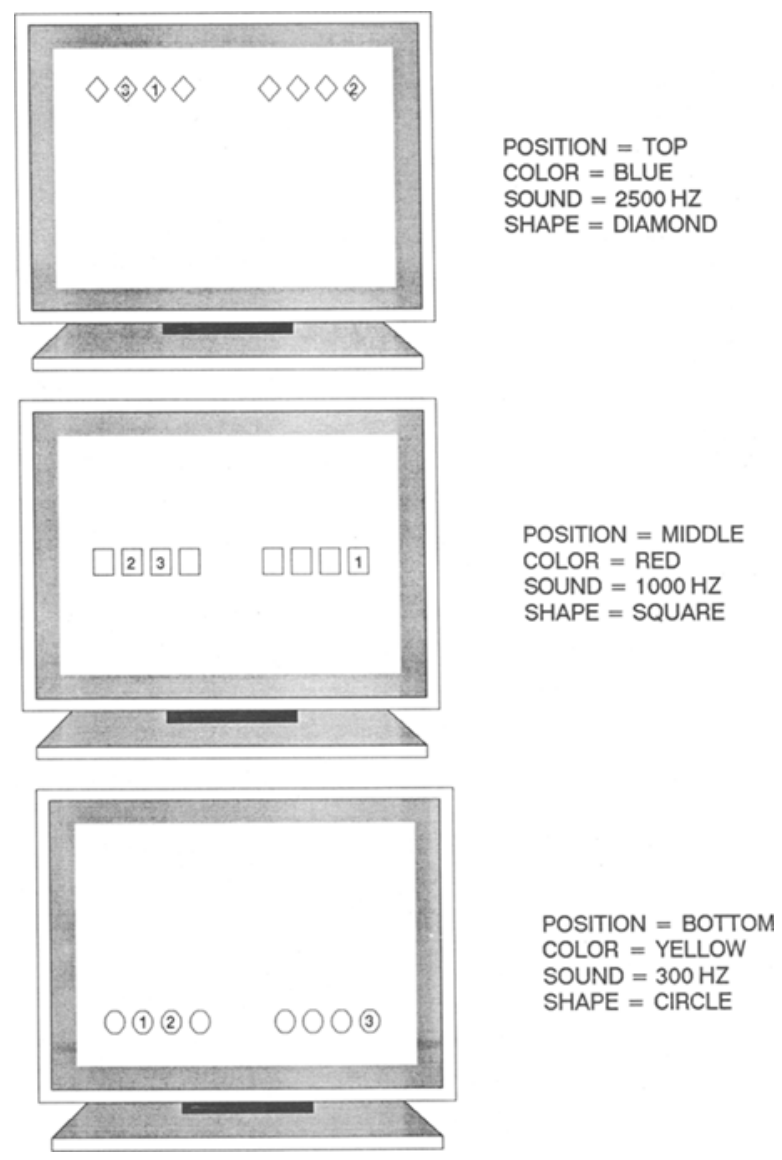

Figure 5. Graphics screen displays for the three sequences used in Experiment 1. Note that each sequence was colored and accompanied by a tone. 


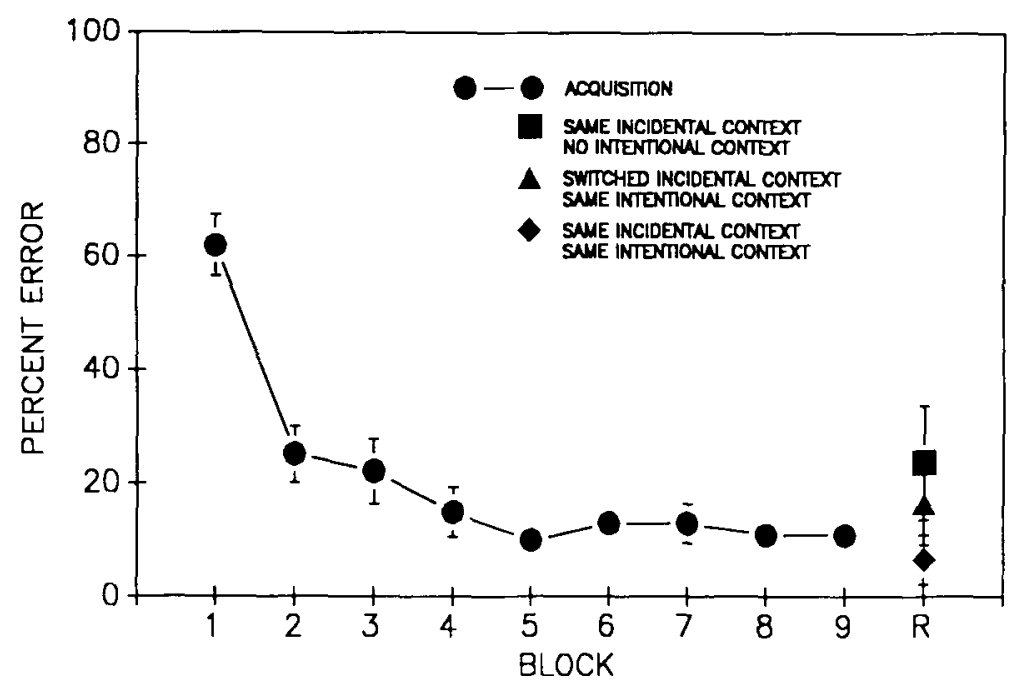

Figure 6. Acquisition and retention performance for the nine acquisition blocks and one retention block in Experiment 2. Note that acquisition performance has been collapsed across retention conditions. Acquisition block represents an average of 12 trials, and the retention block is an average of 3 trials.

ANOVA failed to indicate a main effect of retention condition $[F(2,42)=1.56, p>.05]$.

\section{Discussion}

Reducing the number of keystrokes appears to have led to a reduction in task difficulty. Errors in responding were approximately $10 \%$ at Block 5 and did not fluctuate throughout the remaining blocks of acquisition. The findings from Experiment 2 indicate that reinstating the original learning context led to efficient motor performance comparable with the level achieved at the end of acquisition. This is consistent with the findings from Experiment 1.

The results from Experiment 1 demonstrated that changes in the contextual environment at retention caused a corresponding decrement in motor performance. This led to the conclusion that contextual dependencies had developed. This was not the case for the three-key sequences used in Experiment 2. Changes during retention in the relationship established between the incidental and intentional stimuli during acquisition did not disrupt motor performance.

One explanation suggests that contextual dependencies would be reduced or eliminated because the subject, when faced with learning a relatively easy task, only processes the intentional stimuli. Therefore, switching the incidental stimuli did not disrupt performance, because these stimuli were not processed. In contrast, an alternative proposal maintains that a stronger, more developed association between the intentional stimuli and the task was developed and utilized when a conflict in cues was experienced. The findings from the same intentional/switched incidental retention condition are consistent with both alternatives.

However, when the incidental stimuli were presented alone (no intentional/same incidental retention condition), the two alternatives would predict different outcomes. The position advocating that incidental stimuli are not processed during acquisition would predict that performance cannot be sustained in the absence of the intentional stimuli. This was not the case. The subjects were capable of performing each of the keypressing sequences in the presence of incidental stimuli alone without the aid of the intentional stimuli. This finding indicates that, during acquisition, associations developed between the incidental stimuli and the sequences. This finding is more consistent with the explanation that, given the opportunity, subjects develop a direct association between the incidental stimuli and the task that is capable of sustaining performance. The fact that they could verbalize these associations supports this contention. The findings are congruent with Smith's (1988) suggestion for verbal skills, which states that greater task proficiency will reduce contextual dependencies.

\section{EXPERIMENT 3}

Experiment 1 indicated that contextual reinstatement was extremely important for successful performance of the four-key task. In contrast, in Experiment 2 the need to reestablish the learning context for the three-key task was found to be substantially diminished. These findings suggest that difficulty mediates the development of contextual dependencies during the acquisition of motor tasks. Experiment 3 was conducted in an attempt to extend the findings from the previous experiments in two ways.

First, since the proposed mediating role of difficulty was based on a cross-experiment examination of data, it is important to provide a within-experiment demonstration of the contextual dependency effect. For this reason, in Experiment 3 we included the same intentional/switched incidental and the same intentional/same incidental reten- 
tion conditions for both the three- and the four-key sequences. Since little empirical evidence is available on the contextual dependency development during motor skill learning, the inclusion of these conditions offered the opportunity to demonstrate the reproducibility of the effects demonstrated in Experiments 1 and 2.

Second, retention performance of both four- and threekey sequences was assessed under conditions in which neither intentional nor incidental stimuli manipulated during acquisition were available. This test was incorporated through the inclusion of a retention condition in which subjects were required to free-recall the sequences at the conclusion of the acquisition phase. If in their performances on the four-key sequences subjects are highly reliant on reestablishing the original learning context at retention, free recall would be expected to be significantly poorer than it would be when part or all of the original acquisition context was reinstated. However, since it was proposed that the subjects' performances on the three-key sequences would be less dependent on the need for reinstatement of the learning context at retention, little, if any, aid from intentional or incidental stimuli should have been necessary for successful retention performance. If this should be the case, free recall of the three-key sequences would not differ from the retention performance exhibited in the presence of the entire acquisition context.

\section{Method}

Subjects. The subjects were 72 undergraduate students from Texas A\&M University who received class credit for their participation. None of these subjects had participated in Experiment 1 or 2.

Apparatus. The apparatus was identical to that in Experiments 1 and 2.

Procedures. The procedures were identical to those in Experiments 1 and 2 for the same intentional/same incidental and same intentional/switched incidental retention context conditions. Another condition was added, in which the subjects experienced a no intentional/no incidental context at retention. This condition consisted of a free recall test in which the subjects were requested to execute the sequences that they had learned during the acquisition period. At the conclusion of the experiment, the verbal reports from subjects who had experienced this retention condition were used to assess their knowledge of both intentional and incidental information. All other aspects of Experiment 3 were consistent with Experiments 1 and 2.

\section{Results}

Performance was evaluated in terms of percent correct responses. Acquisition and retention for both the threeand four-key sequences are illustrated in Figure 7, as a function of acquisition block. Note that acquisition performance has been collapsed across retention context conditions.

Acquisition. A sequence $\times$ retention condition $\times$ acquisition block ANOVA with repeated measures on the last factor revealed a main effect of sequence $[F(1,66)=$ $81.09, p<.05]$ and acquisition block $[F(8,528)=96.63$, $p<.05]$. In addition, the sequence $\times$ acquisition block interaction $[F(8,528)=12.59, p<.05]$ was significant. Subsequent post hoc analysis revealed that mean percent error for the three-key sequences was lower than that for the four-key sequences for all acquisition blocks except Acquisition Block 9. All other main effects and interactions were not significant.

Retention. A sequence $\times$ retention condition ANOVA revealed main effects of sequence $[F(1,66)=23.89$, $p<.05]$ and retention condition $[F(2,66)=23.79$, $p<.05]$. In addition, the sequence $\times$ retention condition interaction $[F(2,66)=12.78, p<.05]$ was significant. A subsequent simple main effects analysis for the three-key sequences across retention condition $[F(2,33)$ $=.74, p>.05]$ was not significant. This indicates that

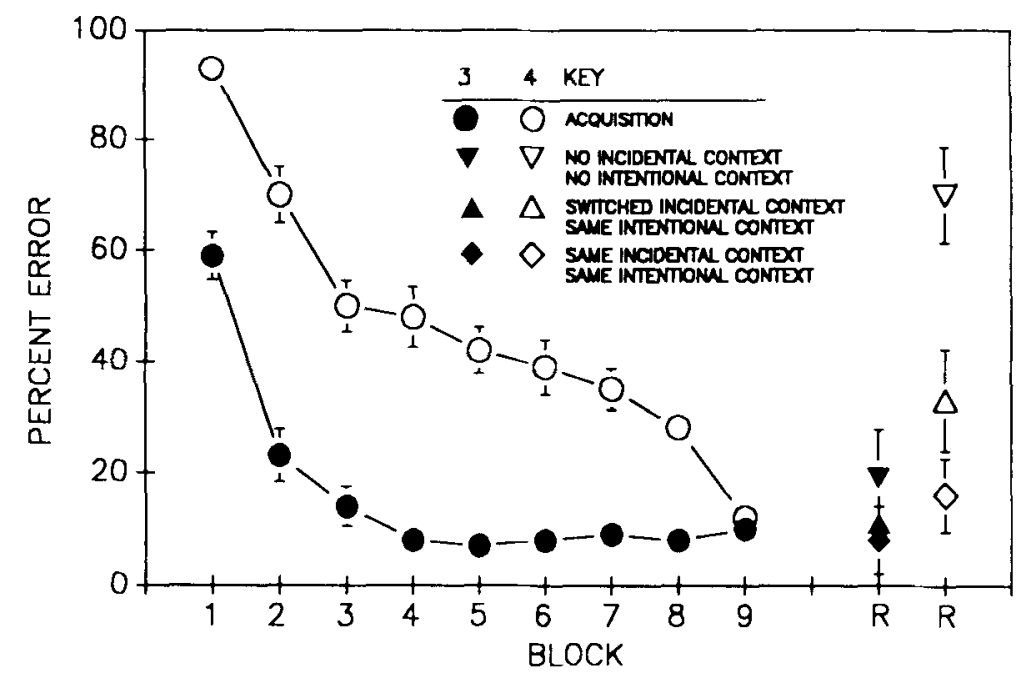

Figure 7. Acquisition and retention performance for the three- and four-key sequences during the nine acquisition blocks and one retention block in Experiment 3. Note that acquisition performance has been collapsed across retention conditions. Acquisition block represents an average of 12 trials, and the retention block is an average of 3 trials. 
the mean percent error for the three three-key sequences did not differ across retention conditions. However, the simple main effects analysis for the four-key sequences across retention condition $[F(2,33)=13.18, p<.05]$ was significant. Duncan's New Multiple Range Test indicated that the mean percent error for the same intentional/same incidental retention condition $(M=16 \%)$ was significantly less than the mean percent error for the same intentional/switched incidental retention condition $(M=$ $33 \%$ ). The latter condition, in turn, exhibited less mean percent error than did the no intentional/no incidental retention context condition $(M=70 \%)$.

\section{Discussion}

Experiment 3 provided a within-experiment replication of the results obtained in Experiments 1 and 2. These findings support the proposal that the development of contextual dependencies during motor skill learning are indeed mediated by difficulty. As in Experiment 1, when a disruption in the environmental context established in acquisition for the four-key task was encountered at retention, error increased beyond that evident when the acquisition context was reinstated at the time of retention. As predicted, this was not the case for retention performance of the three-key sequences.

Findings from the no intentional/no incidental retention condition (free recall) substantiate the conclusions drawn from Experiments 1 and 2. These data verified that reproduction of the four-key sequences was extremely difficult in an environment in which the intentional and incidental stimuli experienced during acquisition were not made available. In contrast, production of the three-key sequences was less susceptible to the contextual dependencies apparent for the four-key sequences. Retrieving and/or executing the three-key sequences was, in effect, relatively context-independent. Individuals apparently had established memorial representations for the three-key sequences that could be directly assessed without the intervention of the manipulated contextual stimuli. However, it should be noted that, when requested, almost every individual was able to provide a precise report of each aspect of the intentional stimuli (key numbers) and the incidental stimuli (colors, sounds, screen position, and key shape), as well as to provide the appropriate relationship with each of the three-key sequences. This suggests that very specific contextual information accompanied the necessary task-specific information in memory. As expected, the verbal reports of the individuals learning the four-key sequences were considerably less accurate and complete.

The memorial associations proposed for the three-key sequences are characterized in Figure 8. Note that the memory network incorporates a bidirectional connection between the tasks and the intentional and incidental cues. This intimates that both intentional and incidental contextual information can be generated from production of the tasks. This was clearly demonstrated in the verbal reports of the subjects. Both performance on the free recall test

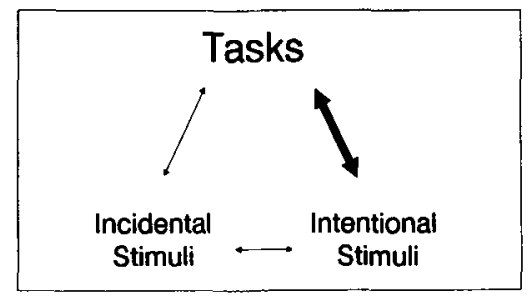

Figure 8. Diagram depicting the proposed associational memory network developed for the three-key sequences.

and the verbal reports obtained from the individuals who acquired the four-key sequences were consistent with the relatively incomplete associational memory network proposed subsequent to Experiment 1 (see Figure 3).

Examination of the relative retention performance of the no intentional/same incidental condition ( $75 \%$ error) from Experiment 1 and the no intentional/no incidental condition (70\% error) from Experiment 3 raises questions regarding the relationship between the incidental stimuli and the tasks depicted in Figure 3. At first glance, this relationship appears suspect, because these conditions led to similar retention performances. Therefore, it could be argued that in the absence of any intentional stimuli, the incidental stimuli have no influence on the performance of the tasks. In light of this, the presence of the direct link between the incidental information and the tasks needs to be reconsidered. ${ }^{3}$

This alternative suggests that correct responses in the no intentional/same incidental retention condition in Experiment 1 were a function of the memorability of the sequences rather than directed by the incidental stimuli. If this were true, subjects would be expected to produce sequences at retention without regard for the incidental information that was currently displayed. However, reexamination of the data from Experiment 1 revealed that this was not the case. Subjects on $25 \%$ of the retention trials produced a response that correctly corresponded to the incidental stimuli presented. Moreover, when the subjects made an error, it rarely consisted of their executing a correct sequence in response to an inappropriate set of incidental stimuli. We interpret this as evidence contrary to the suggestion that subjects use sequence memorability to direct recall.

\section{GENERAL DISCUSSION}

The present set of experiments was an initial attempt to address the development and role of contextual dependencies that evolve during motor skill learning. The findings from these experiments indicate that as the difficulty of the acquired tasks increases, so does the potential for the development of contextual dependencies. Individuals learning the four-key sequences relied particularly on the presence, during retention, of the intentional and incidental context that had been established during acquisition. If the relationship between these stimuli was disrupted by the elimination of one or both sets of stimuli, retention 
performance suffered. Furthermore, verbal reports obtained from individuals acquiring the four-key sequences revealed that their knowledge of the relationships between the contextual stimuli and the sequences was incomplete and inaccurate.

However, subjects' performance of the three-key sequences was proficient even in circumstances in which the contextual environment was different from that in acquisition (Experiments 2 and 3). Moreover, subjects were capable of executing the tasks and could subsequently verbalize the appropriate intentional and incidental stimuli that were associated with each sequence (Experiment 3 ). This suggests that a very comprehensive associational network developed between the contextual cues (both intentional and incidental) and each of the sequences. Taken together, these data from Experiments 1 and 3 suggest that as difficulty increases a less comprehensive associational memory network is established, which, in turn, results in the occurrence of contextual dependencies.

The development of the proposed memory network begins with the initial instructions that lead the learner to associate the intentional stimuli with each of the tasks being acquired. After subsequent practice, an association develops between the intentional and incidental stimuli. This relationship can support successful performance of the tasks if the relationship remains consistent. If this relationship is disrupted, however, as in the case of the fourkey sequences in Experiment 1, performance deteriorates.

Only when difficulty is decreased does the association between the incidental stimuli and the tasks appear to emerge. This is highlighted by the precise verbal reports obtained from subjects who learned the three-key sequences in Experiments 2 and 3. In this situation, the contextual dependencies were reduced to the extent that either intentional or incidental stimuli alone or in combination could sustain performance. This perspective suggests that both intentional and incidental stimuli are maintained as part of the internal representation of the tasks. However, both types of stimuli can be used independently as retrieval cues, with the intentional stimuli representing the stronger, more developed relationship with the tasks.

Although it appears that the memory network developed during acquisition of the three-key sequences is reasonably elaborate, note that the contextual dependencies that are evident during acquisition of the four-key sequences are reduced for the three-key sequences but not necessarily eliminated. More specifically, although in Experiment 2 no statistical difference in performance was apparent for different retention conditions, the relative ordering of these conditions was the same as that demonstrated for the four-key sequences in Experiment 1 . It is possible, therefore, that the sensitivity of the dependent measure (percent correct responses) used in the present experiments was not sufficient to highlight additional dependencies.

Lintern (1985) has suggested that the availability of additional cues can aid task performance during acquisition. However, if dependencies develop, performance can be negatively affected should these additional sources be withdrawn. This perspective is not far removed from the "crutch" analogy recently advocated by a number of motor theorists for the role of knowledge of results for motor skill learning (Salmoni, Schmidt, \& Walter, 1984; Winstein \& Schmidt, 1990). This suggests that learners are often inclined to develop an overreliance on particular types of information through practice. Should this information be removed at some subsequent stage of learning, severe performance decrements would be exhibited.

It would be interesting to speculate that the associations that develop between each of the stimuli and the tasks leading to the subsequent dependencies exhibited represent the progression that occurs throughout the learning of a motor task. Intuitively, one might assume that as practice continues, the development of the memory network incorporating the associations between the intentional stimuli, incidental stimuli, and the tasks would become more comprehensive. If this is the case, contextual dependencies should be diminished as practice progresses. The potential contributions of practice to contextual dependencies awaits future experimentation.

The present findings offer some interesting implications for present theoretical perspectives on skilled motor learning. Recently, Lee (1988) advocated that the commonalities of the processing activities engaged in by the learner during acquisition and retention may prove to be an important consideration for the learning of motor information. This was offered in terms of the transfer-appropriate processing perspective (Morris, Bransford, \& Franks, 1977), according to which learning can be enhanced if the processing engendered during a period of task acquisition is compatible with that required at subsequent tests of retention. One would assume that the extent to which particular processing activities can be reengaged would be dependent on the availability of that information in the environment. This being the case, it appears that examination of the structure of the environment in which such processing might occur should demand some further experimental attention.

Furthermore, the present findings also pose an interesting question regarding the proposal of Salmoni et al. (1984), who reemphasized the use of transfer designs to highlight and distinguish transient performance effects from relatively permanent learning effects. These authors stressed the need to examine all experimental conditions in subsequent test situations at a consistent level of the manipulated independent variable. They contended that only after the temporary performance effects had diminished could an assessment of the permanent learning effects be determined.

This procedure has been used in a variety of areas of study in the motor domain, including contextual interference (Lee \& Magill, 1983), relative frequency of knowledge of results (Winstein \& Schmidt, 1990), summary knowledge of results (Schmidt, Young, Swinnen, \& Shapiro, 1989), and massed versus distributed practice (Stelmach, 1969). Any differences identified at reten- 
tion are usually attributed to the particular experimental manipulation being investigated. However, the present findings suggest that the change in the contextual environment experienced in some experimental conditions may be sufficient to account for some of the retention differences commonly demonstrated.

\section{REFERENCES}

DULSKY, S. G. (1935). The effect of a change of background on recall and relearning. Journal of Experimental Psychology, 18, 725-740.

Eich, J. E., Weingartner, H., Stillman, R. C., \& Gillin, J. C. (1975). State dependent accessibility of retrieval cues in the retention of a categorized list. Journal of Verbal Learning \& Verbal Behavior, 14, 408-417.

LEE, T. D. (1988). Transfer-appropriate processing: A framework for conceptualizing practice in motor learning. In $\mathrm{O}$. G. Meijer \& $\mathrm{K}$. Roth (Eds.), Complex movement behaviour: "The" motor-action controversy (pp. 201-215). Amsterdam: Elsevier Science (North-Holland).

LEE, T. D., \& MAgill, R. A. (1983). The locus of the contextual interference in motor skill acquisition. Joumal of Experimental Psychology: Learning, Memory, \& Cognition, 9, 730-746.

LiNTERN, G. (1985). Context and dependency effects in skill acquisition. In R. Eberts \& C. G. Eberts (Eds.), Trends in ergonomics/human factors II (pp. 249-256). Amsterdam: Elsevier Science (NorthHolland).

Morris, C. D., Bransford, J. D., Franks, J. J. (1977). Levels of processing versus transfer appropriate processing. Journal of Verbal Learning \& Verbal Behavior, 16, 519-533.

REED, H. J. (1931). The influence of a change of condition upon the amount recalled. Journal of Experimental Psychology, 14, 632-649.

SAlmoni, A. W., Schmid, R. A., \& Walter, C. B. (1984). Knowledge of results and motor learning: A review and critical appraisal. Psychological Bulletin, 95, 355-386.

Schmidt, R. A., Young, D. E., Swinnen, S., Shapiro, D. C. (1989). Summary knowledge of results for skill acquisition: Support for the guidance hypothesis. Journal of Experimental Psychology: Learning, Memory, \& Cognition, 15, 352-359.

SCHWARTZ, B., \& BARSKY, S. F. (1977). The home advantage. Social Forces, 55, 641-661.

SILVA, J. M., ANDREW, A., RICHEY, S. (1983). Game location and basketball performance variation. Paper presented at the annual conference of the North American Society for the Psychology of Sport and Physical Activity, East Lansing, MI.

SMIth, S., \& GuThrIe, E. R. (1924). General psychology in terms of behavior. New York: Appleton-Century.

SMITH, S. M. (1986). Environmental context-dependent recognition memory using a short-term memory task for input. Memory \& Cognition, 14, 347-354.

SMITH, S. M. (1988). Environmental context-dependent memory. In G. M. Davies and D. M. Thomson (Eds.), Memory in context: Context in memory (pp. 13-34). New York: Wiley.

Smith, S. M., Glenberg, A., BJoRK, R. A. (1978). Environmental context and human memory. Memory \& Cognition, 6, 342-353.

Stelmach, G. E. (1969). Efficiency of motor learning as a function of intertrial rest. Research Quarterly, 40, 198-202.

Underwood, B. J., HaM, M., E Ekstrand, B. R. (1962). Cue selection in paired-associate learning. Joumal of Experimental Psychology, 64, 405-409.

VARCA, P. (1980). An analysis of home and away game performance of male college basketball teams. Journal of Sport Psychology, 2, 245-257.

VELA, E. (1984). Memory as a function of environmental context. Paper presented at the 30th annual meeting of the Southwest Psychological Association, New Orleans, LA.

Watkins, M. J., Ho, E., \& Tulving, E. (1976). Context effects in recognition memory. Journal of Verbal Leaming \& Verbal Behavior, 15, 505-518.

Winstein, C. J., \& SChmidT, R. A. (1990). Reduced frequency of knowledge of results enhances motor skill learning. Journal of Experimental Psychology: Learning, Memory, \& Cognition, 16, 677-691.

\section{NOTES}

1. This classification of environmental stimuli may not be as appropriate for experiments in the verbal domain. Verbal experiments have been primarily aimed at the influence of ambient stimuli present during the entire acquisition experiences rather than stimuli that might become associated with a specific task. An exception comprises the verbal studies done with cued recall. For example, Dulsky (1935) presented CVC pairs on different colored cards during acquisition and either switched or kept the same color of the card on which the cue (stimulus CVC) was presented during retention. In this case, the stimulus CVC would be considered the intentional stimulus and the color of the card would be the incidental stimulus.

2. The correlation between the performance on the last block of acquisition and the retention block with switched incidental stimuli was significant $(r=.52, p<.05)$. Correlations for the groups in which the incidental and intentional stimuli were reinstated and the incidental stimuli were presented alone failed to reach significance $(r=-.19$, $p>.05$, and $r=.25, p>.05$, respectively).

3. We thank Tim Lee for pointing out this potential change to the proposed memory network.

(Manuscript received June 1, 1990; revision accepted for publication January 11, 1991.) 